\title{
El perfil emprendedor y la intensidad competitiva del mercado como predictores de supervivencia en microempresas mexicanas
}

\author{
The entrepreneurial profile and the competitive intensity of the market \\ as predictors of survival in mexican microenterprises
}

Francisco Javier Segura Mojica*

Tecnológico Nacional de México/Instituto Tecnológico de San Luis Potosí, México

Recibido el 16 de julio de 2018; aceptado el 8 de mayo de 2019

Disponible en Internet el: 21 de mayo de 2019

\section{Resumen}

En este trabajo se muestran los resultados de una investigación realizada en 93 microempresas de la zona conurbada de San Luis Potosí-Soledad de Graciano Sánchez, México, con el objetivo de encontrar factores que influyen sobre sus posibilidades de supervivencia a largo plazo. Se utilizó un modelo de regresión logística logit para estimar la forma en que la intensidad competitiva de un sector, la localización geográfica, el perfil del emprendedor, la realización de estudios de mercado y la concentración geográfica de competidores influyen sobre las probabilidades de sobrevivencia o cierre de un negocio. Entre los hallazgos relevantes destacan una mayor importancia relativa de las variables perfil del emprendedor y estudio de mercado.

Código JEL: C35, L26, M13

Palabras clave: Supervivencia de microempresas; Perfil emprendedor; Intensidad competitiva

\footnotetext{
* Autor para correspondencia

Correo electrónico recursosmx@yahoo.com (F. J. Segura Mojica).

La revisión por pares es responsabilidad de la Universidad Nacional Autónoma de México. 


\begin{abstract}
This paper shows the results of an investigation carried out in 93 micro companies of the conurbation of San Luis Potosí-Soledad de Graciano Sanchez, Mexico, with the objective of finding factors that influence their chances of long-term survival. A logit logistic regression model was used to estimate the way in which the competitive intensity of a sector, the geographical location, the profile of the entrepreneur, the performance of market studies and the geographical concentration of competitors influence the probabilities of business survival. Among the relevant findings, a greater relative importance of the variables profile of the entrepreneur and market study stand out.
\end{abstract}

JEL Code: C35, L26, M13

Keywords: Micro companies survival; Entrepreneurial profile; Competitive intensity

\title{
Introducción
}

Conocer los factores que influyen sobre la supervivencia de un negocio es una cuestión esencial para gestionar adecuadamente proyectos de emprendimiento y programas de impulso a la creación de empresas. Los primeros cinco años de vida de un negocio suelen ser los que representan mayores retos, pues durante ese período es cuando ocurre la mayor proporción de cierre de negocios; en México por ejemplo, el 33\% de los negocios desaparecen durante el primer año de vida, y sólo el 35\% sobrevive después de cinco años a partir de su creación (INEGI, 2018).

A nivel global, la baja rentabilidad es el factor que más se menciona como la principal causa de cierre de los negocios (Global Entrepreneurship Monitor, 2014), seguida por los motivos personales y los problemas para obtener financiamiento (Tabla 2).

Sin embargo, es posible que bajo los problemas de rentabilidad y financiamiento existan factores como la forma de tomar decisiones, la percepción del mercado y el estilo de gestión que asume el emprendedor o directivo del negocio.

La pregunta de investigación que da origen al presente trabajo es ¿Existe alguna relación estadística entre la probabilidad de sobrevivencia de un proyecto de emprendimiento y el valor de algunos factores determinantes de su desempeño, tales como el perfil del emprendedor, la intensidad competitiva del sector en el que incursiona, las características de localización de la empresa, la cantidad de competidores en el lugar donde se estableció y la implementación de estudios de mercado? 
Tabla 1

Supervivientes por cada 100 negocios que ingresan a la actividad económica por sector económico, según edad

\begin{tabular}{ccccc}
\hline $\begin{array}{c}\text { Edad de los } \\
\text { negocios }\end{array}$ & Manufacturero & Comercio & $\begin{array}{c}\text { Servicios privados no } \\
\text { financieros }\end{array}$ & Total \\
\hline 0 & 100 & 100 & 100 & 100 \\
1 & 70 & 66 & 68 & 67 \\
5 & 40 & 33 & 36 & 35 \\
10 & 30 & 23 & 26 & 25 \\
15 & 24 & 16 & 20 & 19 \\
20 & 20 & 12 & 15 & 15 \\
25 & 17 & 9 & 12 & 11 \\
\hline
\end{tabular}

Fuente: INEGI (2018). Esperanza de vida de los negocios, con datos de los Censos Económicos 1989, 1994, 1999, 2004, 2009 y 2014.

Tabla 2

Principales razones para el cierre de un negocio.

\begin{tabular}{lc}
\hline Causa & $\%$ \\
\hline Oportunidad de vender el negocio & $3.9 \%$ \\
El negocio no era rentable & $30.7 \%$ \\
Problemas para obtener financiamiento & $13.2 \%$ \\
Otro trabajo u oportunidad de negocio & $9.6 \%$ \\
Salida planificada & $3.4 \%$ \\
Retiro & $4.4 \%$ \\
Motivos personales & $17.9 \%$ \\
Un incidente & $3.1 \%$ \\
Gobierno, impuestos, burocracia & $4.3 \%$ \\
Otro & $9.6 \%$ \\
\hline
\end{tabular}

Fuente: Elaboración propia con información del Global Entrepreneurship Monitor (2015) APS Global Individual Level Data. 


\section{Revisión de la literatura}

En México, una microempresa se define como la unidad de negocio cuya plantilla laboral es de 10 trabajadores o menos y cuyas ventas no superan los cuatro millones de pesos (Diario Oficial de la Federación, 2011). En México existen 5.3 millones de microempresas, de las cuáles $11 \%$ corresponden al sector industrial, 50\% al comercial, $37 \%$ al sector servicios y $2 \%$ a otras actividades económicas (INEGI, 2015).

La fuerte concentración de microempresas en los sectores comercial y de servicios se traduce en mercados altamente fragmentados y competidos, lo que parece influir en su baja rentabilidad, rápida salida del mercado, crecimiento marginal, y dificultades para desarrollar ventajas competitivas sostenibles.

Desde la perspectiva del paradigma estructura-conducta-resultado (Zou y Cavusgil, 2002; Ruppenthal y Bausch, 2009), podríamos deducir que la sobrevivencia de una unidad de negocio está en función del grado de concentración de oferta y demanda, diferenciación de sus productos y barreras de entrada; estos tres factores configuran la estructura del mercado, así como de las elecciones estratégicas que las empresas realicen para el uso de sus recursos (conducta).

Como Porter (2008) observa, es necesario comprender la estructura subyacente del sector en el que el emprendedor incursiona, tomando como referencia las cinco fuerzas competitivas: amenaza de nuevos entrantes, amenaza de productos o servicios sustitutos, poder de negociación de los proveedores, poder de negociación de los compradores y rivalidad entre los competidores existentes.

Siguiendo el supuesto de Porter en el sentido de que una mayor intensidad de las fuerzas competitivas se traduce en bajos retornos de las inversiones, la incursión en sectores intensamente competidos equivaldría a mayores posibilidades de cierre prematuro del negocio.

\section{Mercados e interacción espacial: la importancia de la localización}

Si al elegir el giro del negocio el emprendedor decide el entorno competitivo al que se enfrentará, al elegir la localización de su empresa, traduce a factores espaciales algunas de las fuerzas competitivas del mercado y puede potenciar las ventajas y/o neutralizar las amenazas.

Los pequeños comercios son especialmente sensibles al factor localización. Sin embargo, los negocios enfocados a la manufactura también pueden verse afectados por la ubicación de sus instalaciones, particularmente por cuestiones relacionadas con su cadena de suministros. Este elemento se potencia cuando se considera que los micro negocios suelen depender de la fidelidad de clientelas delimitadas en espacios geográficos relativamente pequeños. Por tanto, elegir una ubicación conveniente, estimando el potencial del lugar donde planea instalarse el proyecto de negocio, puede ser de vital importancia para su éxito o fracaso. 
Tabla 3

Fuerzas competitivas que dan forma a la estrategia y factores que las integran

\begin{tabular}{|c|c|c|}
\hline Fuerza competitiva & Factor & Item \\
\hline \multirow{7}{*}{$\begin{array}{l}\text { Amenaza de nuevos } \\
\text { entrantes }\end{array}$} & Economías de escala por el lado de la oferta & AE1 \\
\hline & Beneficios de escala por el lado de la demanda & AE2 \\
\hline & Bajo costos para los clientes por cambiar de proveedor & AE3 \\
\hline & Requisitos de capital & AE4 \\
\hline & Ventajas de actores establecidos independientemente del tamaño & AE5 \\
\hline & Acceso desigual a canales de distribución & AE6 \\
\hline & Políticas gubernamentales restrictivas & AE7 \\
\hline \multirow{6}{*}{$\begin{array}{l}\text { Poder de los provee- } \\
\text { dores }\end{array}$} & Concentración & PP1 \\
\hline & Baja dependencia del sector para sus ingresos & PP2 \\
\hline & Los participantes asumen los costos de cambiar de proveedor & PP3 \\
\hline & Los proveedores ofrecen productos diferenciados & PP4 \\
\hline & No existe un substituto para lo que ofrece el proveedor & PP5 \\
\hline & Amenaza de integrarse al sector en forma más avanzada & PP6 \\
\hline \multirow{7}{*}{$\begin{array}{l}\text { Poder de los compra- } \\
\text { dores }\end{array}$} & Hay pocos compradores o compran en volúmenes grandes & PC1 \\
\hline & Los productos del sector son estantarizados o no se diferencían entre sí & PC2 \\
\hline & Los clientes asumen bajos costos por cambiar de proveedor & PC3 \\
\hline & Los compradores pueden integrarse hacia atrás en la cadena & PC4 \\
\hline & El grupo de proveedores busca bajar costos & PC5 \\
\hline & $\begin{array}{l}\text { La calidad del producto/servicio del cliente no se ve afectada por la calidad de } \\
\text { los productos del sector }\end{array}$ & PC6 \\
\hline & El producto del sector afecta poco los costos del comprador & PC7 \\
\hline \multirow{2}{*}{$\begin{array}{l}\text { Amenaza de produc- } \\
\text { tos sustitutos }\end{array}$} & Ofrecen una relación atractiva de precio y servicio & APS 1 \\
\hline & El costo para el comprador para cambiar por el substituto es bajo. & APS2 \\
\hline \multirow{6}{*}{$\begin{array}{l}\text { Rivalidad entre com- } \\
\text { petidores existentes }\end{array}$} & Hay varios competidores similares en tamaño y potencia & RCE1 \\
\hline & El crecimiento del sector es lento & RCE2 \\
\hline & Las barreras de salida son altas & RCE3 \\
\hline & Las empresas no son capaces de entender bien sus señales mutuamente & RCE3 \\
\hline & Los rivales son altamente comprometidos con el negocio o aspiran a ser líderes & RCE4 \\
\hline & Hay competencia de precios & RCE5 \\
\hline
\end{tabular}

Fuente: Elaboración propia con base en Porter, 2007 
Grasland (2004) apunta que el cálculo del potencial de un lugar se basa en una hipótesis de interacción espacial: la probabilidad de llegada de los clientes se da en relación con la distancia, la cual se refiere a una medida de accesibilidad que apunta a evaluar la variación de la cantidad de oportunidades de relación en función de la posición.

Garrocho (2003), puntualiza que la localización de un negocio puede determinar el éxito o fracaso de un proyecto de negocios. En este sentido, sintetiza los postulados de la Teoría de la Interacción Espacial (TIE) de la siguiente manera: el número de consumidores que atrae una unidad comercial depende simultáneamente de dos fuerzas: los costos de transporte para que los consumidores accedan al negocio, y lo atractivo de los negocios.

Dicho de otra forma, en tanto mayor sea la distancia o dificultad para acceder a un establecimiento, mayor será el costo para el consumidor y menor el incentivo para acceder al mismo. Sin embargo, este factor interactúa con el grado de atracción que el negocio ejerza sobre el cliente. El autor considera que a pesar de que la localización es un factor de importancia estratégica para el éxito de un negocio, suele definirse a partir de la intuición y el conocimiento práctico del empresario, más que de análisis fundamentados. Por otra parte, en las decisiones sobre localización también pueden incidir factores tales como conveniencia personal del propietario (por cercanía con su domicilio o por contar con un local).

\section{Conducta emprendedora y decisiones estratégicas}

En contraste con el enfoque estructura-conducta-resultado, cuyo énfasis está en el análisis de los factores externos a la organización, la teoría de los recursos y las capacidades (Grant, 1991, citado por Ibarra y Suárez, 2002) sugiere que el desarrollo de los factores internos es la clave para el desarrollo de la estrategia y la construcción de ventajas competitivas.

El contexto de las microempresas, especialmente las enfocadas al comercio, es de una fuerte escasez de recursos y capacidades limitadas, por lo que el perfil del emprendedor-directivo y más específicamente, la conducta emprendedora, podrían considerarse como la capacidad base o esencial, desde donde se puede definir la estrategia y por lo tanto, las posibilidades de sobrevivencia y crecimiento de este tipo de empresas (figura 1).

Shane y Venkataraman (2000), y más adelante Pedrosa (2015) definen la conducta emprendedora como "el descubrimiento, evaluación y explotación de oportunidades que permitan introducir nuevos productos, servicios, procesos, formas de organización o mercado en la sociedad". 


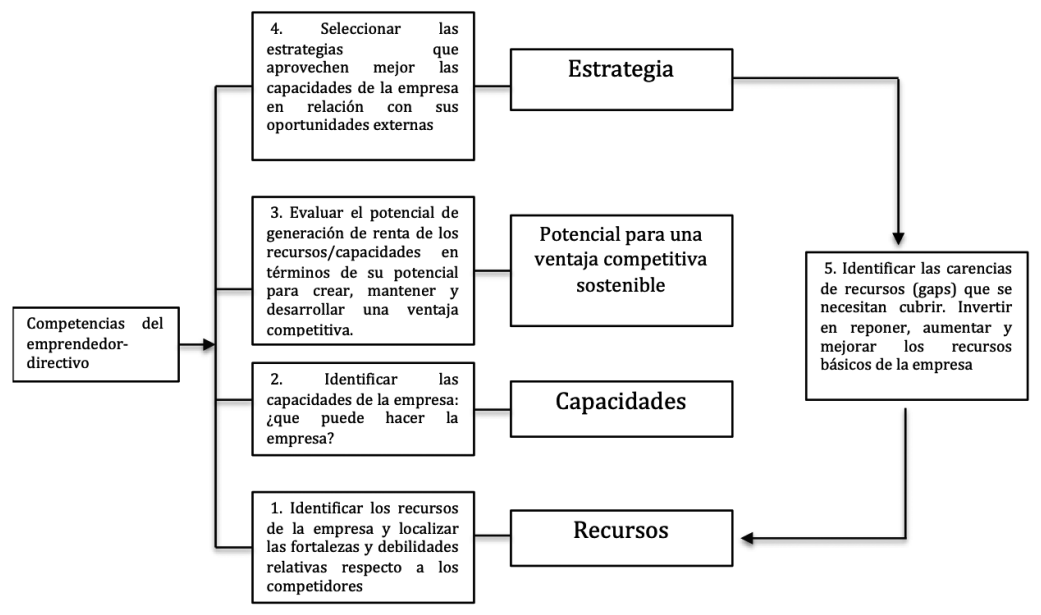

Figura 1. Las competencias del emprendedor y su relación práctica con el análisis estratégico en el marco de la teoría de los recursos y las capacidades.

Fuente: Adaptado de Grant, 1991; Ibarra y Suárez 2002

Si bien la señal distintiva del emprendedor es la creación de unidades de negocio, el aprendizaje, la innovación, la incertidumbre y el riesgo son componentes que parecen estar fuertemente vinculados con el perfil del emprendedor.

Sobre las motivaciones para emprender, se habla de que existe un perfil del emprendedor, cuyos rasgos principales se relacionan con las actitudes. Formichella (2002) señala que la diferencia entre el emprendedor y el individuo común está determinada por una propensión diferente a asumir riesgos, enfrentar problemas, descubrir oportunidades ocultas, crear redes de comunicación, formar equipos y vencer los temores.

Existen diferentes formas de organizar los rasgos, y por lo tanto, de definir el perfil del emprendedor. Entre los primeros acercamientos al tema, se encuentra el trabajo de Hornaday (1982), quien propuso una lista de 42 rasgos. Sánchez (2017) tomando como referentes a Covin \& Slevin (1989); Cromie (2000); y Vecchio (2003), reduce la lista a cuatro rasgos que son Locus de Control, Autoeficacia, Riesgo y Proactividad.

Para los fines de la presente investigación, se toma como base la clasificación propuesta por Pedrosa (2015), quien toma como base el modelo de Rauch y Frese (2007), que considera una visión global de la conducta emprendedora, e integra los rasgos generales con otros más específicos propios del comportamiento emprendedor. De esta forma, a los rasgos ya mencionados en el párrafo anterior se añaden el de Motivación al Logro, Innovación, Optimismo y 
Tolerancia al Estrés. Con base en este modelo se desarrolló el cuestionario "Test Adaptativo Informatizado para la evaluación de la personalidad emprendedora (TAI)", el cuál se utilizó como instrumento de recolección de información en el presente estudio.

Molina (2009), tomando como base la propuesta de Bruyat y Julien (2001) propone una clasificación de los empresarios, según el nivel de riesgo que son propensos a asumir: empresarios de reproducción, de imitación, de valorización o de aventura. Esta clasificación nos puede ayudar a entender por qué no todos los emprendedores logran que su proyecto se convierta en una empresa de mayor tamaño o incluso, capaz de sobrevivir. Sin embargo, también abre otras interrogantes, como por ejemplo si existen modelos psicológicos que puedan ayudarnos a caracterizar la conducta de los emprendedores; y si además de los rasgos de personalidad existen valores o actitudes que puedan estar especialmente relacionados con el emprendimiento.

\section{Materiales y métodos}

Se realizó una investigación cuantitativa de alcance explicativo y diseño transversal. Para el análisis estadístico se utilizó el software XLSTAT 2016.

El objetivo de la investigación es comprender la forma en que interactúan las variables perfil del emprendedor, intensidad competitiva del sector, localización y concentración de negocios similares, con la variable sobrevivencia del negocio.

La hipótesis se planteó de la siguiente forma:

Ho: La diferencia entre las medias de las submuestras G1 y G2 para las variables Perfil emprendedor, Estudio de mercado, Intensidad Competitiva del Mercado, Potencial de la localización y Concentración de competidores directos es igual a cero.

H1: La diferencia entre las medias de las submuestras G1 y G2 para las variables Perfil emprendedor, Estudio de mercado, Intensidad Competitiva del Mercado, Potencial de la localización y Concentración de competidores directos es diferente de cero. 
Tabla 4

Operacionalización de las variables

\begin{tabular}{|c|c|c|}
\hline Variable & Dimensiones & Indicadores \\
\hline \multirow{8}{*}{ Perfil del emprendedor } & Motivación de logro & Puntuación en la dimensión correspondiente del TAI \\
\hline & Toma de riesgos & Puntuación en la dimensión correspondiente del TAI \\
\hline & Innovación & Puntuación en la dimensión correspondiente del TAI \\
\hline & Autonomía & Puntuación en la dimensión correspondiente del TAI \\
\hline & Locus de control interno & Puntuación en la dimensión correspondiente del TAI \\
\hline & Autoeficacia & Puntuación en la dimensión correspondiente del TAI \\
\hline & Tolerancia al estrés & Puntuación en la dimensión correspondiente del TAI \\
\hline & Optimismo & Puntuación en la dimensión correspondiente del TAI \\
\hline \multirow{5}{*}{$\begin{array}{l}\text { Intensidad competitiva } \\
\text { del sector }\end{array}$} & Amenaza de nuevos entrantes & Número de factores de la tabla 3 observados \\
\hline & Poder de los proveedores & Número de factores de la tabla 3 observados \\
\hline & Poder de los compradores & Número de factores de la tabla 3 observados \\
\hline & $\begin{array}{l}\text { Amenaza de productos sus- } \\
\text { titutos }\end{array}$ & Número de factores de la tabla 3 observados \\
\hline & $\begin{array}{l}\text { Rivalidad entre los competi- } \\
\text { dores existentes }\end{array}$ & Número de factores de la tabla 3 observados \\
\hline \multirow{7}{*}{$\begin{array}{l}\text { Potencial de la locali- } \\
\text { zación }\end{array}$} & Tráfico & Flujo de transeúntes \\
\hline & \multirow{3}{*}{ Atracción acumulada } & Microlocalización: En esquina, en un centro comercial, etc. \\
\hline & & Nivel socioeconómico de la zona \\
\hline & & Densidad de población \\
\hline & Compatibilidad & Existencia de negocios afines o complementarios en la zona \\
\hline & \multirow{2}{*}{ Accesibilidad } & Estacionamiento \\
\hline & & Vialidad \\
\hline $\begin{array}{l}\text { Concentración de com- } \\
\text { petidores directos }\end{array}$ & $\begin{array}{l}\text { Cantidad de competidores } \\
\text { directos en la zona }\end{array}$ & Número de competidores directos en un radio de 100 metros \\
\hline Estudio de mercado & $\begin{array}{l}\text { Realización de estudio de } \\
\text { mercado }\end{array}$ & Realización de estudio de mercado \\
\hline
\end{tabular}

Fuente: Elaboración propia 
Se obtuvo una muestra de 100 empresas localizadas en el Estado de San Luis Potosí, de las cuáles 93 proporcionaron datos válidos. De estas empresas, el 70\% se mantiene en funcionamiento y tiene por lo menos 5 años de operaciones, mientras que el $30 \%$ cesaron sus operaciones en el transcurso del año 2017. Esta condición nos permitió comparar los valores de las dos submuestras.

Tabla 5

Segmentación de la muestra por sector y subsector de actividad económica

\begin{tabular}{|c|c|c|c|c|c|}
\hline Sector & $\%$ & Subsector & $\%$ & $\% \mathrm{G} 1$ & $\% \mathrm{G} 2$ \\
\hline \multirow{9}{*}{ Comercio } & \multirow{9}{*}{49} & $\begin{array}{l}\text { Comercio al por menor de abarrotes, alimentos, } \\
\text { bebidas, hielo y tabaco }\end{array}$ & 26 & 15 & 11 \\
\hline & & Comercio al por menor de artículos de papelería, para & & & \\
\hline & & el esparcimiento y otros artículos de uso personal & 12 & 9 & 3 \\
\hline & & Comercio al por menor de artículos de ferretería, & & & \\
\hline & & tlapalería y vidrios & 2 & 2 & 0 \\
\hline & & Comercio al por menor de productos textiles, bisute- & & & \\
\hline & & ría, accesorios de vestir y calzado & 7 & 4 & 3 \\
\hline & & Comercio al por menor de artículos para el cuidado & & & \\
\hline & & de la salud & 2 & 2 & 0 \\
\hline \multirow{4}{*}{$\begin{array}{l}\text { Industrias manu- } \\
\text { factureras }\end{array}$} & \multirow{4}{*}{28} & Elaboración de productos de panadería y tortillas & 14 & 10 & 4 \\
\hline & & Impresión e industrias conexas & 7 & 7 & 0 \\
\hline & & Fabricación de muebles, excepto oficina y estantería & 2 & 1 & 1 \\
\hline & & Otras industrias manufactureras & 5 & 5 & 0 \\
\hline \multirow{4}{*}{$\begin{array}{l}\text { Servicios privados } \\
\text { no financieros }\end{array}$} & \multirow{4}{*}{23} & $\begin{array}{l}\text { Reparación y mantenimiento de automóviles y ca- } \\
\text { miones }\end{array}$ & 7 & 4 & 3 \\
\hline & & Otros servicios/Lavanderías y tintorerías & 2 & 2 & 0 \\
\hline & & Salones y clínicas de belleza & 2 & 2 & 0 \\
\hline & & Servicios de preparación de alimentos y bebidas & 12 & 7 & 5 \\
\hline
\end{tabular}

Nota: G1 = Muestra 1 (empresas que se mantienen en operación); G2=Muestra 2 (empresas que cerraron durante 2017)

Fuente: Elaboración propia 
El método de muestreo fue aleatorio simple, tomando como universo la base de datos del Directorio Nacional de Unidades Económicas (DENUE) del Instituto Nacional de Estadística y Geografía (INEGI). Fueron seleccionadas empresas con 10 trabajadores o menos, por lo que corresponden a la categoría de microempresas. Los instrumentos de recolección de información fueron aplicados de manera presencial, entrevistando al fundador-propietario o principal directivo en funciones. Esta condición es relevante debido a que uno de los elementos analizados es el modelo psicológico del emprendedor. Cabe señalar que el empleo de un solo informante es conveniente para reducir los errores relacionados con la existencia de diferentes perspectivas de un mismo fenómeno.

\section{Escalas de medida}

Se realizaron tres mediciones. En primer lugar se aplicó el cuestionario "Test Adaptativo Informatizado para la evaluación de la personalidad emprendedora (TAI)" formulado por Pedrosa (2015). Este cuestionario consta de 107 ítems organizados en 8 categorías: motivación de logro, toma de riesgos, innovación, autonomía, autoeficacia, tolerancia al estrés, locus de control interno y optimismo. Como resultado se obtuvo un puntaje acumulado al que llamaremos Perfil emprendedor, que sintetiza el grado en que un individuo posee los rasgos y actitudes asociados a la implementación exitosa de un negocio. Para aportar evidencias de validez de contenido, se recurrió al juicio de expertos que analizaron la relevancia y representatividad de los ítems obteniéndose un puntaje medio de 3.85 en una escala de 1 a 5 para valorar la pertinencia de los ítems (Pedrosa, 2015), cuyos índices de discriminación se distribuyeron entre 0.24 y 0.66 y sus pesos factoriales entre 0.24 y 0.73 mostrando, además, unos adecuados índices de ajuste (Tabla 6).

La fiabilidad total de la batería, estimada mediante el coeficiente alfa de Cronbach para datos ordinales fue de 0.96 .

También se utilizó el Análisis Factorial Exploratorio de segundo orden para determinar la existencia de un factor general denominado "Perfil Emprendedor" relacionado con los nueve rasgos específicos. La tabla 7 muestra la existencia de dicho factor que explica el $49 \%$ de la varianza con niveles de ajuste adecuados. 
Tabla 6

Propiedades psicométricas de las subescalas del TAI

\begin{tabular}{lcccccccc} 
& \multicolumn{9}{c}{ Pesos fac- } & Var. \\
& $\mathrm{n}$ & $\mathrm{ID}$ & $\alpha$ & toriales & GFI & RMSR & ET & Exp. \\
\hline Motivación de logro & 15 & $0.37-0.63$ & 0.88 & $0.4-0.66$ & 0.99 & 0.04 & 0.054 & $36 \%$ \\
Toma de riesgos & 15 & $0.24-0.59$ & 0.84 & $0.3-0.61$ & 0.98 & 0.05 & 0.054 & $29 \%$ \\
Innovación & 15 & $0.33-0.61$ & 0.85 & $0.37-0.61$ & 0.98 & 0.053 & 0.053 & $31 \%$ \\
Autonomía & 14 & $0.26-0.54$ & 0.82 & $0.27-0.67$ & 0.97 & 0.067 & 0.054 & $28 \%$ \\
Locus de control interno & 9 & $0.27-0.61$ & 0.85 & $0.43-0,73$ & 0.99 & 0.043 & 0.053 & $43 \%$ \\
Autoeficacia & 20 & $0.28-0.66$ & 0.98 & $0.27-0.62$ & 0.98 & 0.045 & 0.054 & $30 \%$ \\
Tolerancia al estrés & 14 & $0.29-0.57$ & 0.81 & $0.24-0.68$ & 0.92 & 0.1 & 0.054 & $27 \%$ \\
Optimismo & 11 & $0.40-0.62$ & 0.85 & $0.4-0,72$ & 0.99 & 0.046 & 0.054 & $38 \%$ \\
& & & & & & & & \\
\hline
\end{tabular}

Nota: ID=Indices de discriminación; GFI=Indice de bondad de ajuste; RMSR=Raíz media cuadrática de los residuales; ET=Error tipico; Var. Exp.=Varianza explicada

Fuente: Pedrosa, 2015

Tabla 7

Análisis Factorial Exploratorio de las puntuaciones factoriales de cada subescala del TAI

Perfil emprendedor

Autoeficacia

Motivación de logro

Innovación

Locus de control interno

Optimismo

Autonomía

Toma de riesgos

Tolerancia al estrés

Varianza explicada

GFI

RMSR (Error tipico)
0.92

0.88

0.74

0.63

0.6

0.55

0.55

0.49

49.07

0.97

$0.07(0.06)$

Nota: GFI= Indice de bondad de ajuste; RMSR (Raíz Media cuadrática de los residuales)

Fuente: Pedrosa, 2015 
A este cuestionario se añadió la pregunta ¿Ha realizado investigación de mercado al inicio o durante el desarrollo de su proyecto de emprendimiento?, con lo que se evaluó la variable Investigación de mercado.

Para evaluar el potencial de la localización, se diseñó un cuestionario denominado "Plantilla para evaluar los factores de ubicación de un negocio" (ver tabla 8). Para validar el contenido de este instrumento, se tomó como referencia el modelo propuesto por De Juan (2005), quien propone las dimensiones tráfico, atracción acumulada, compatibilidad y accesibilidad como criterios para decidir la ubicación de puntos de venta. El análisis de confiabilidad de este cuestionario obtuvo un coeficiente Alfa de Cronbach de 0.706

Tabla 8

Plantilla para evaluar los factores de ubicación de un negocio

\begin{tabular}{|c|c|c|c|}
\hline Dimensión & Indicador & Escala de medida & \\
\hline \multirow{4}{*}{ Tráfico } & \multirow{4}{*}{ Flujo de transeúntes } & Muy alto (Más de 50 personas por minuto) & 4 \\
\hline & & Alto (De 30 a 50 personas por minuto) & 3 \\
\hline & & Medio (De 10 a 30 personas por minuto) & 2 \\
\hline & & Bajo (Menos de 10 personas por minuto) & 1 \\
\hline \multirow{11}{*}{ Atracción acumulada } & \multirow{4}{*}{ Micro localización } & Dentro de un mercado/plaza comercial & 4 \\
\hline & & En la parte exterior de un mercado o plaza comercial & 3 \\
\hline & & En una esquina & 0 \\
\hline & & A mitad de cuadra & 1 \\
\hline & \multirow{3}{*}{$\begin{array}{l}\text { Nivel socioeconómi- } \\
\text { co de la zona }\end{array}$} & Alto & 3 \\
\hline & & Medio & 2 \\
\hline & & Escasos recursos & 1 \\
\hline & \multirow{4}{*}{$\begin{array}{l}\text { Densidad de pobla- } \\
\text { ción }\end{array}$} & Muy alta (más de 4185 personas por AGEB) & 4 \\
\hline & & Alta (De 2870 a 4185 personas por AGEB) & 3 \\
\hline & & Media (de 1952 a 2870 personas por AGEB) & 2 \\
\hline & & Baja (menos de 1952 personas por AGEB) & 1 \\
\hline
\end{tabular}




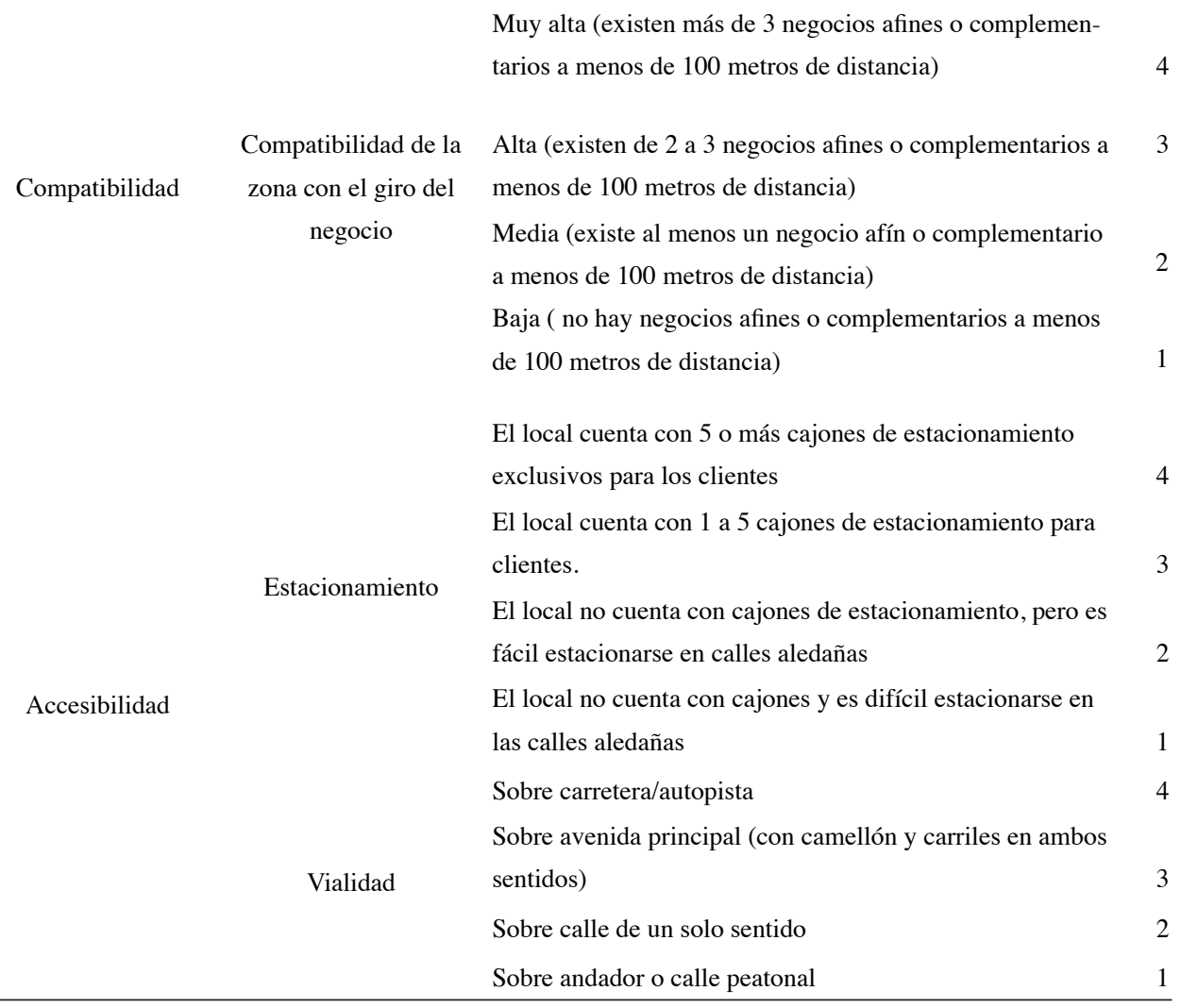

Fuente: Elaboración propia.

La validez del constructo se determinó mediante el análisis factorial exploratorio, utilizando para la extracción de factores el método de máxima verosimilitud. Como se muestra en las tablas 9 y 10, se confirma que existen cuatro factores que explican el 56\% de la varianza acumulada. La extracción de cuatro factores es consistente con lo propuesto por el modelo teórico.

Como resultado se obtiene un puntaje que indicaría qué tan ventajosa es la localización del lugar. Cabe aclarar que no se evaluaron factores tales como el comercio electrónico, redes sociales y páginas web, los cuáles influyen sobre la accesibilidad del negocio sin los costos de transporte para que los consumidores accedan al negocio, y visualicen lo atractivo de los negocios.

A este cuestionario se le añadió la pregunta "Número de competidores directos en un radio de 100 metros a partir de la localización”, con lo que se evaluó la variable Concentración de competidores directos. 


\begin{tabular}{|c|c|c|c|c|c|c|c|c|}
\hline & \multicolumn{4}{|c|}{ Matriz de configuración inicial } & \multicolumn{4}{|c|}{$\begin{array}{l}\text { Matriz de configuración después de rotación } \\
\text { Varimax }\end{array}$} \\
\hline & F1 & $\mathrm{F} 2$ & F3 & F4 & D1 & D2 & D3 & D4 \\
\hline Micro localización & 0.516 & -0.520 & -0.098 & 0.106 & 0.106 & 0.251 & 0.660 & 0.217 \\
\hline Vialidad & 0.499 & -0.231 & 0.090 & 0.090 & 0.305 & 0.160 & 0.393 & 0.213 \\
\hline $\begin{array}{l}\text { Compatibilidad de la zona con el giro } \\
\text { del negocio }\end{array}$ & 0.382 & -0.320 & -0.368 & 0.296 & 0.002 & -0.092 & 0.680 & 0.023 \\
\hline Nivel socioeconómico de la zona & 0.556 & 0.474 & -0.305 & 0.059 & 0.322 & -0.613 & 0.185 & 0.342 \\
\hline Estacionamiento & 0.538 & -0.035 & -0.289 & -0.309 & 0.004 & -0.150 & 0.300 & 0.598 \\
\hline Densidad de población & 0.733 & 0.375 & 0.481 & 0.293 & 0.972 & -0.109 & 0.080 & 0.180 \\
\hline Flujo de transeúntes & 0.599 & 0.063 & 0.143 & -0.477 & 0.276 & 0.077 & 0.008 & 0.727 \\
\hline Variabilidad (\%) & 27.081 & 13.930 & 8.892 & 6.661 & 15.405 & 11.773 & 15.154 & 14.172 \\
\hline
\end{tabular}

Los valores en negrita corresponden para cada variable al factor para el cual el coseno cuadrado es el mayor.

Fuente: Elaboración propia

Tabla 10

Prueba de bondad de ajuste del Análisis Factorial Exploratorio de los Indicadores de Potencial de Localización

\begin{tabular}{lcccc}
\hline Chi-cuadrado (Valor observado) & $\begin{array}{c}\text { Chi-cuadrado } \\
\text { (Valor crítico) }\end{array}$ & GL & valor-p & alfa \\
\hline 5.327 & 5.991 & 2 & 0.070 & 0.05 \\
Nota: & & & & \\
\hline
\end{tabular}

H0: 4 factores comunes son suficientes para describir los datos.

Ha: Se necesitan más factores para describir los datos.

Puesto que el valor-p calculado es mayor que el nivel de significación alfa $=0.05$, no se puede rechazar la hipótesis nula $\mathrm{HO}$.

Fuente: Elaboración propia

Finalmente, se utilizó la Tabla 3 del presente artículo, basada en el modelo de Fuerzas Competitivas de Porter, para determinar qué tan intensa es la competitividad del sector en el que han incursionado los proyectos de emprendimiento. Cada factor da lugar a una respuesta $\mathrm{Si} / \mathrm{No}$, con lo que se obtienen variables binarias $1 / 0$ que indican la presencia o ausencia de cada una de las características asociadas a la fuerzas competitivas. Este cuestionario obtuvo un coeficiente Alfa de Cronbach de 0.897.

Como fundamento para validar el contenido de la lista, se toma como referencia a Porter 2008, quien cita cada uno de los ítems incluidos en la lista como factores de intensidad compe- 
titiva, de tal forma que este indicador es resultante de la sumatoria de los factores observados en los diferentes sectores y subsectores incluidos en la muestra.

La validez de constructo se realizó mediante la técnica de Análisis Factorial Exploratorio para el conjunto de respuestas a los ítems de los cuestionarios, examinando la estructura factorial de las puntuaciones e identificando las fuentes de variación en las medidas observadas. Se trataba de confirmar, por un lado, la existencia de un constructo subyacente que agrupase a la mayor parte de los ítems y que explicase el razonamiento sobre el intervalo de confianza.

Para el instrumento "Fuerzas competitivas y factores que las integran", la extracción obtuvo 5 factores de los cuáles el primero explica el $76 \%$ de la variabilidad. Este número de factores es consistente con lo indicado por el modelo teórico de Porter.

Tabla 11

Análisis Factorial Exploratorio del cuestionario "Fuerzas Competitivas y factores que las integran"

\begin{tabular}{|c|c|c|c|c|c|c|c|c|c|c|}
\hline \multirow[b]{2}{*}{ Item } & \multicolumn{5}{|c|}{ Matriz de configuración inicial } & \multicolumn{5}{|c|}{$\begin{array}{c}\text { Matriz de configuración después de rotación } \\
\text { varimax }\end{array}$} \\
\hline & $\mathrm{F} 1$ & F2 & F3 & $\mathrm{F} 4$ & F5 & D1 & D2 & D3 & D4 & D5 \\
\hline ANE1 & 0.517 & 0.497 & -0.039 & -0.097 & -0.258 & 0.484 & 0.572 & -0.098 & -0.081 & 0.117 \\
\hline ANE2 & 0.261 & 0.678 & 0.033 & 0.257 & -0.080 & 0.225 & 0.574 & -0.271 & 0.307 & 0.232 \\
\hline ANE3 & -0.101 & 0.711 & -0.008 & 0.209 & 0.222 & -0.127 & 0.457 & -0.088 & 0.436 & 0.431 \\
\hline ANE4 & -0.264 & 0.451 & -0.121 & 0.621 & -0.089 & -0.253 & 0.375 & -0.261 & 0.631 & -0.100 \\
\hline ANE5 & -0.127 & -0.118 & -0.694 & -0.597 & 0.190 & -0.045 & 0.027 & 0.935 & -0.163 & -0.029 \\
\hline ANE5 & -0.069 & 0.247 & -0.507 & -0.404 & -0.131 & -0.030 & 0.427 & 0.549 & -0.124 & -0.055 \\
\hline ANE7 & 0.992 & -0.058 & -0.092 & 0.020 & 0.006 & 0.997 & -0.008 & -0.027 & -0.026 & 0.010 \\
\hline PP1 & 0.364 & 0.337 & 0.323 & -0.104 & 0.521 & 0.305 & -0.034 & -0.110 & 0.062 & 0.723 \\
\hline PP2 & 0.992 & -0.058 & -0.092 & 0.020 & 0.006 & 0.997 & -0.008 & -0.027 & -0.026 & 0.010 \\
\hline PP3 & 0.156 & 0.230 & 0.731 & 0.168 & -0.268 & 0.049 & 0.142 & -0.786 & -0.200 & 0.178 \\
\hline PP4 & -0.166 & 0.389 & 0.661 & -0.222 & 0.419 & -0.271 & -0.019 & -0.305 & -0.152 & 0.807 \\
\hline PP5 & 0.253 & 0.171 & 0.550 & -0.383 & -0.402 & 0.154 & 0.261 & -0.409 & -0.647 & 0.159 \\
\hline PP6 & 0.305 & 0.755 & 0.173 & -0.021 & -0.206 & 0.237 & 0.698 & -0.292 & -0.003 & 0.326 \\
\hline PC1 & -0.992 & 0.058 & 0.092 & -0.020 & -0.006 & -0.997 & 0.008 & 0.027 & 0.026 & -0.010 \\
\hline $\mathrm{PC} 2$ & -0.274 & -0.532 & -0.309 & -0.060 & -0.071 & -0.206 & -0.335 & 0.331 & -0.071 & -0.438 \\
\hline PC3 & -0.080 & 0.728 & -0.246 & 0.138 & -0.459 & -0.088 & 0.872 & -0.078 & 0.198 & -0.112 \\
\hline PC4 & -0.240 & 0.282 & -0.315 & 0.776 & 0.166 & -0.187 & 0.137 & -0.093 & 0.887 & -0.127 \\
\hline
\end{tabular}




\begin{tabular}{lcccccccccc}
\hline PC5 & -0.168 & 0.662 & -0.389 & -0.238 & 0.128 & -0.156 & 0.596 & 0.422 & 0.175 & 0.321 \\
PC6 & 0.992 & -0.058 & -0.092 & 0.020 & 0.006 & 0.997 & -0.008 & -0.027 & -0.026 & 0.010 \\
PC7 & 0.026 & -0.421 & -0.004 & 0.038 & -0.240 & 0.046 & -0.233 & -0.053 & -0.166 & -0.388 \\
APS1 & -0.199 & 0.502 & -0.436 & -0.190 & -0.308 & -0.177 & 0.685 & 0.318 & 0.020 & -0.102 \\
APS2 & 0.283 & 0.281 & -0.333 & 0.556 & 0.344 & 0.329 & 0.088 & 0.036 & 0.756 & 0.099 \\
RCE1 & 0.992 & -0.058 & -0.092 & 0.020 & 0.006 & 0.997 & -0.008 & -0.027 & -0.026 & 0.010 \\
RCE2 & 0.992 & -0.058 & -0.092 & 0.020 & 0.006 & 0.997 & -0.008 & -0.027 & -0.026 & 0.010 \\
RCE3 & -0.992 & 0.058 & 0.092 & -0.020 & -0.006 & -0.997 & 0.008 & 0.027 & 0.026 & -0.010 \\
RCE4 & 0.992 & -0.058 & -0.092 & 0.020 & 0.006 & 0.997 & -0.008 & -0.027 & -0.026 & 0.010 \\
RCE5 & 0.307 & 0.502 & 0.052 & -0.563 & 0.261 & 0.258 & 0.341 & 0.245 & -0.265 & 0.649 \\
RCE6 & -0.245 & 0.736 & -0.074 & -0.419 & 0.082 & -0.283 & 0.625 & 0.249 & -0.083 & 0.500 \\
Variabili- & & & & & & & & & & \\
dad (\%) & 32.458 & 18.092 & 10.526 & 9.946 & 5.403 & 32.031 & 14.476 & 10.165 & 9.859 & 9.894 \\
& & & & & & & & & & \\
\hline
\end{tabular}

Fuente: Elaboración propia

Tabla 12

Prueba de bondad de ajuste del Análisis Factorial Exploratorio del instrumento Fuerzas competitivas y factores que los integran

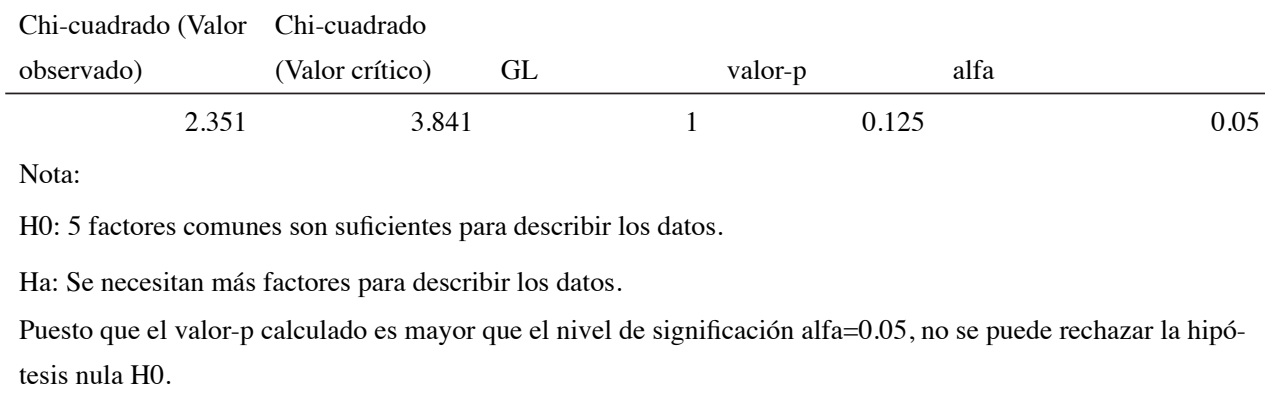

Fuente: Elaboración propia

Como se muestra en la tabla 11, se confirma que existen cinco factores que explican el $76.4 \%$ de la varianza acumulada. La extracción de cinco factores es consistente con lo propuesto por el modelo teórico. Finalmente, se realizó un análisis factorial de segundo orden sobre las 
puntuaciones factoriales de cada una de las subescalas, encontrándose que existe un factor que explica el $44 \%$ de la varianza, al cuál podríamos identificar como intensidad competitiva.

\section{Análisis estadístico}

En primer lugar se aplicaron pruebas de hipótesis z y t para determinar si existe diferencia de medias entre las submuestras G1 y G2.

Tabla 13

Pruebas z y t para dos muestras

\begin{tabular}{|c|c|c|c|c|c|c|c|c|c|c|}
\hline Variable & $\begin{array}{c}\text { Sub-mues- } \\
\text { tra }\end{array}$ & Media & $\begin{array}{c}\text { Desviación } \\
\text { Estandar }\end{array}$ & $\mathrm{z}$ & $\begin{array}{l}\text { | z | Valor } \\
\text { crítico }\end{array}$ & $\begin{array}{c}\text { p-value } \\
\text { (bilateral) }\end{array}$ & $\mathrm{t}$ & $\begin{array}{c}|\mathrm{t}| \\
\text { Valor } \\
\text { crítico }\end{array}$ & $\begin{array}{c}\text { p-value } \\
\text { (bilate- } \\
\text { ral) }\end{array}$ & alfa \\
\hline $\begin{array}{l}\text { Perfil em- } \\
\text { prendedor }\end{array}$ & $\mathrm{G} 2$ & 3.535 & 0.490 & -2.722 & 1.960 & 0.006 & -2.678 & 1.986 & 0.009 & 0.05 \\
\hline \multirow{3}{*}{$\begin{array}{l}\text { Estudio de } \\
\text { mercado }\end{array}$} & G1 & 3.840 & 0.510 & & & & & & & \\
\hline & G2 & 0.179 & 0.390 & -2.776 & 1.960 & 0.006 & -2.514 & 1.986 & 0.014 & 0.05 \\
\hline & G1 & 0.446 & 0.501 & & & & & & & \\
\hline \multirow[t]{2}{*}{$\begin{array}{l}\text { Intensidad } \\
\text { competitiva }\end{array}$} & G2 & 19.200 & 4.315 & 0.387 & 1.960 & 0.699 & 0.400 & 2.024 & 0.692 & 0.05 \\
\hline & G1 & 18.600 & 4.048 & & & & & & & \\
\hline Potencial de & & & & & & & & & & \\
\hline la localiza- & $\mathrm{G} 2$ & 13.900 & 3.414 & -1.192 & 1.960 & 0.233 & -1.278 & 2.026 & 0.209 & 0.05 \\
\hline ción & G1 & 15.345 & 2.967 & & & & & & & \\
\hline Concentra- & & & & & & & & & & \\
\hline $\begin{array}{l}\text { ción de los } \\
\text { competi- }\end{array}$ & G2 & 2.400 & 0.843 & 0.104 & 1.960 & 0.917 & 0.097 & 2.024 & 0.923 & 0.05 \\
\hline dores & G1 & 2.367 & 0.964 & & & & & & & \\
\hline
\end{tabular}

Fuente: Elaboración propia

Como puede apreciarse, las pruebas z y t muestran que existe diferencia de medias entre las submuestras para las variables Perfil emprendedor y Estudio de mercado, pues el valor 
p bilateral es menor que el nivel de significación alfa. Esto no se cumple para las variables Intensidad competitiva, potencial de la localización y concentración de los competidores.

Posteriormente, con el fin de profundizar en el análisis, se utilizó un modelo de regresión no lineal con respuesta cualitativa, Logit. Este tipo de modelos son aquellos donde la variable dependiente puede ser de naturaleza cualitativa mientras que las variables independientes pueden ser cualitativas, cuantitativas o una mezcla de ambas (Moscote y Rincón, 2012).

La forma general del modelo logit es

$$
\mathrm{E}(\mathrm{y})=\frac{e^{x^{\prime} \beta}}{1+e^{x^{\prime} \beta}}
$$

donde $x$ es el vector de variables independientes y beta es el vector de parámetros. La transformación de logit de la probabilidad pi realiza de la siguiente forma:

$$
\begin{aligned}
\mathrm{E}(\mathrm{y}) & =\frac{1}{1+e^{-x^{\prime} \beta}} \\
\pi \mathrm{i} & =\frac{1}{1+e^{-x^{\prime} \beta}} \\
1-\pi \mathrm{i} & =\frac{1}{1+e^{x^{\prime} \beta}} \\
\frac{\pi i}{1-\pi i} & =\frac{1+e^{x^{\prime} \beta}}{1+e^{-x^{\prime} \beta}}=e^{x^{\prime} \beta}
\end{aligned}
$$

Finalmente, al tomar el logaritmo natural se obtiene:

$$
\operatorname{Ln}\left(\frac{\pi i}{1-\pi i}\right)=\mathrm{x}^{\prime} \beta
$$


La forma gráfica del modelo logit se muestra en la figura 1, donde se puede observar que los valores de la variable dependiente se encuentran entre 0 y 1.

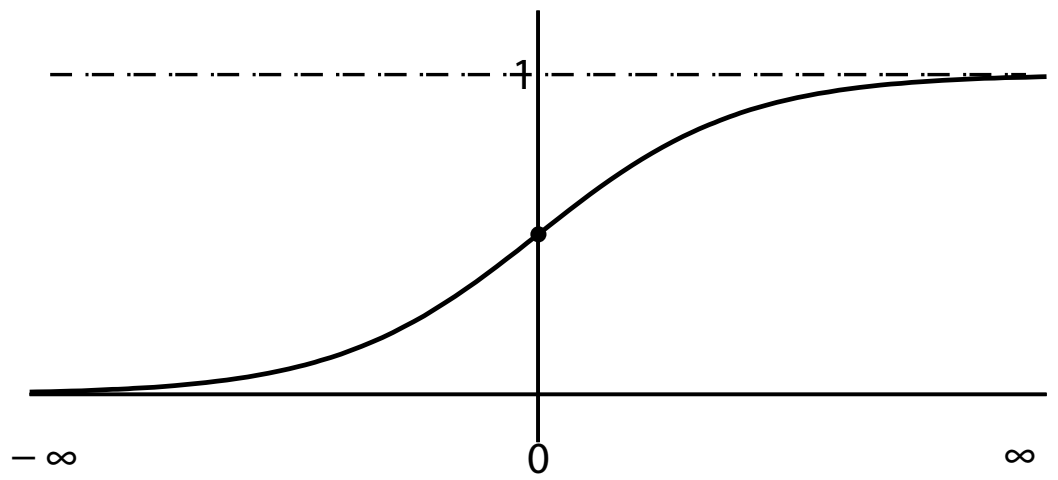

Figura 2. Función Logit

Fuente: Moscote y Rincón, 2012.

La estimación de los parámetros se realizó mediante el método de máxima verosimilitud (Green, 2001, citado por Moscote y Rincón, 2012) obteniéndose los siguientes resultados:

Tabla 14

Estadísticos descriptivos de la variable supervivencia

\begin{tabular}{cccc}
\hline Variable & Categorías & Frecuencias & $\%$ \\
\hline \multirow{2}{*}{ Supervivencia del negocio } & 0 & 28 & 30.108 \\
& 1 & 65 & 69.892 \\
\hline
\end{tabular}

Fuente: Elaboración propia. 
Tabla 15

Estadísticos descriptivos de las variables independientes

\begin{tabular}{|c|c|c|c|c|c|c|c|}
\hline Variable & $\begin{array}{c}\text { Observacio- } \\
\text { nes }\end{array}$ & $\begin{array}{l}\text { Obs. con } \\
\text { datos } \\
\text { perdidos }\end{array}$ & $\begin{array}{c}\text { Obs. sin } \\
\text { datos } \\
\text { perdidos }\end{array}$ & Mínimo & Máximo & Media & $\begin{array}{l}\text { Desv. } \\
\text { típica }\end{array}$ \\
\hline Perfil del emprendedor & 93 & 0 & 93 & 2.337 & 4.830 & 3.748 & 0.521 \\
\hline \multicolumn{8}{|l|}{ Intensidad competitiva del } \\
\hline sector & 93 & 0 & 93 & 11.000 & 23.000 & 18.750 & 2.649 \\
\hline Potencial de la localización & 93 & 0 & 93 & 10.000 & 22.000 & 14.974 & 1.997 \\
\hline Concentración de competi- & & & & & & & \\
\hline dores directos & 93 & 0 & 93 & 1.000 & 4.000 & 2.375 & 0.602 \\
\hline
\end{tabular}

Fuente: Elaboración propia

Tabla 16

Estadísticos de bondad de ajuste

\begin{tabular}{lrr}
\hline Estadístico & Independiente & Completo \\
Observaciones & 93 & 93 \\
\hline Suma de los pesos & 93.000 & 93.000 \\
GL & 92 & 87 \\
-2 Log(Verosimilitud) & 113.790 & 102.909 \\
R2$^{2}$ McFadden) & 0.000 & 0.096 \\
R $^{2}$ Cox and Snell) & 0.000 & 0.110 \\
$\mathrm{R}^{2}$ (Nagelkerke) & 0.000 & 0.156 \\
AIC & 115.790 & 114.909 \\
SBC & 118.322 & 130.104 \\
Iteraciones & 0 & 6 \\
\hline
\end{tabular}

Fuente: Elaboración propia 
Tabla 17

Prueba de la hipótesis nula H0: Y=0.699 (Variable Supervivencia):

\begin{tabular}{lrrr}
\hline Estadístico & GL & Chi-cuadrado & Pr $>\mathrm{Chi}^{2}$ \\
$-2 \log$ (Verosimilitud) & 5 & 10.881 & 0.050 \\
Score & 5 & 10.312 & 0.060 \\
Wald & 5 & 9.185 & 0.100 \\
\hline
\end{tabular}

Fuente: Elaboración propia

Las tablas 16 y 17 nos proporcionan indicadores de la calidad del modelo o calidad del ajuste. En este caso, el valor más significativo es el $\mathrm{Chi}^{2}$ asociado al log (verosimilitud). Como la probabilidad es 0.05 se puede deducir que el modelo proporciona una cantidad significativa de información.

Tabla 18

Parámetros del modelo

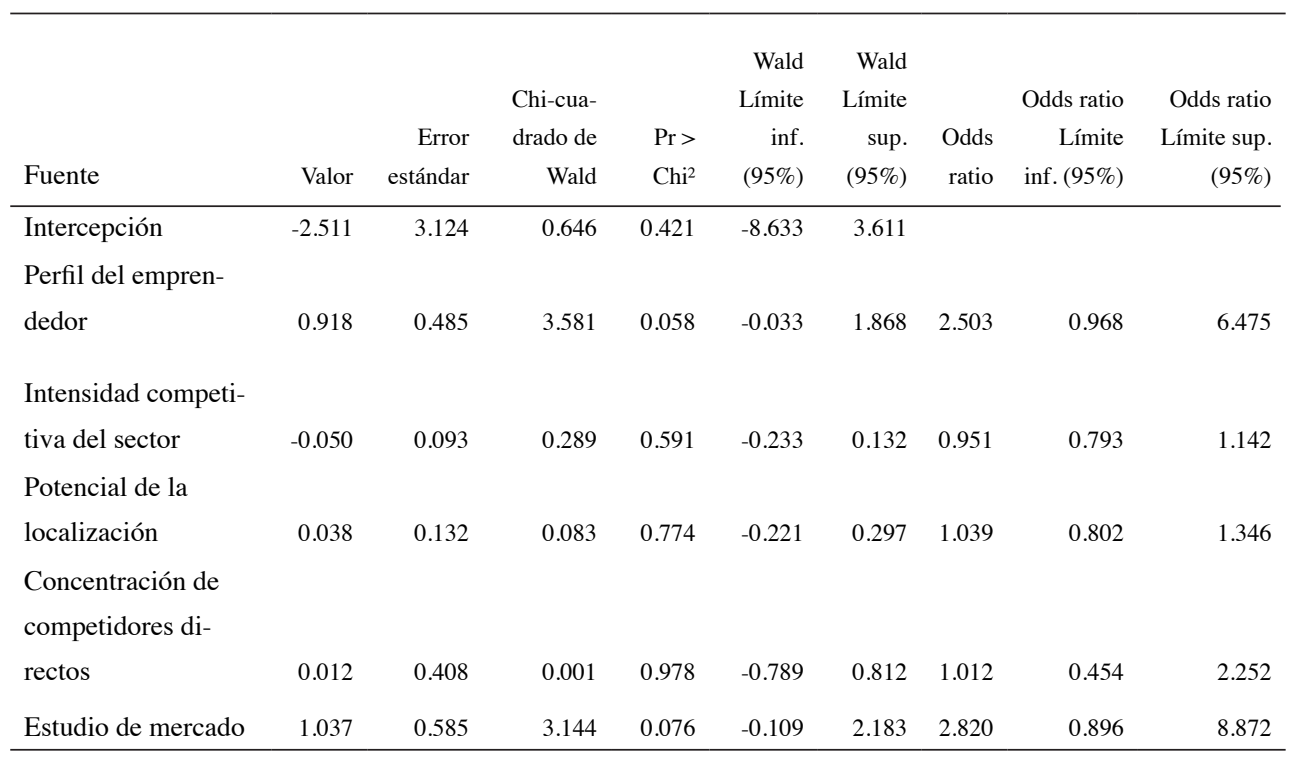

Fuente: Elaboración propia 
La tabla 9 proporciona detalles sobre el modelo, y es útil para comprender el efecto de las diferentes variables sobre las categorías de la variable de respuesta. Muestra una intercepción para cada categoría de la variable de respuesta y un conjunto de coeficientes ya que se supone que se cumplirá la hipótesis de curvas paralelas.

Aquí podemos observar que según la probabilidad asociada a las pruebas de $\mathrm{Chi}^{2}$ de Wald, la variable que más influye sobre la probabilidad de sobrevivencia del negocio es el perfil del emprendedor, seguida en importancia por la realización del estudio de mercado. Esto se confirma al analizar el gráfico de coeficientes estandarizados.

Las odds ratio o razón de oportunidades nos muestran que un negocio para el que se han realizado estudios de mercado tendría 2.8 veces más oportunidad de sobrevivir; mientras que un perfil emprendedor alto significa 2.5 veces más oportunidad de sobrevivir. En la tabla 19 podemos observar que según la probabilidad asociada a las pruebas de $\mathbf{C h i}^{2}$, la variable que más influye es el perfil del emprendedor, seguida en importancia por la realización de un estudio de mercado. La intensidad competitiva del sector tiene un papel significativo, aunque de signo negativo.

\section{Tabla 19}

Coeficientes estandarizados (Variable supervivencia)

\begin{tabular}{lcr}
\hline Fuente & Valor & $\operatorname{Pr}>\mathrm{Chi}^{2}$ \\
Perfil del emprendedor & 0.262 & 0.05 \\
Intensidad competitiva del sector & -0.073 & 0.59 \\
Potencial de la localización & 0.042 & 0.77 \\
Concentración de competidores directos & 0.004 & 0.97 \\
Realizó estudio de mercado & 0.275 & 0.07 \\
\hline
\end{tabular}

Fuente: Elaboración propia.

\section{Ecuación del modelo de supervivencia}

Pred $($ Supervivencia $)=1 /(1+\exp (-(-2.511+0.917 *$ Perfil del emprendedor- $0.05 *$ Intensidad competitiva del sector $+0.037 *$ Potencial de la localización $+0.0115 *$ Concentración de competidores+1.036*Realizó estudio de mercado-1)))

La aplicación del modelo permite apreciar que cuando el perfil del emprendedor es pobre o cuando no se realiza estudio de mercado, las probabilidades de sobrevivencia del negocio decrecen significativamente; una mayor intensidad competitiva impacta negativamente en las probabilidades de sobrevivencia, pero en menor grado. 


\section{Conclusiones}

El análisis estadístico nos permite visualizar la forma en que las características de los emprendedores interactúan con la estructura del mercado como factores determinantes para la supervivencia de los micro negocios.

Las pruebas de hipótesis z y t muestran en principio, que los valores promedio de las submuestras G1 y G2 son significativamente diferentes para las variables Perfil Emprendedor y Estudio de Mercado; sin embargo, estas diferencias no son significativas para el caso de las variables Intensidad competitiva del mercado, Potencial de la Localización y Concentración de competidores directos.

Este hallazgo se confirma al utilizar el modelo de regresión logística Logit, como puede apreciarse en la tabla 19 y en la ecuación del modelo, donde se muestra que los factores que ejercen mayor influencia sobre las posibilidades de supervivencia de un negocio, son el perfil del emprendedor y la realización del estudio de mercado.

Este hallazgo es consistente con el supuesto de Grant que conecta el desempeño exitoso del negocio con las competencias del emprendedor, tales como identificar recursos, capacidades, oportunidades para lograr ventaja y rentabilidad, así como el diseño de estrategias que relacionen los recursos y capacidades con las oportunidades externas. También parece ser consistente con el modelo propuesto por Morales et al (2015), en el que el perfil del emprendedor interactúa la capacidad de establecer conexiones con los clientes, dando forma a los atributos pivote que permiten permanecer en el mercado.

Según se aprecia en los resultados del modelo Logit, la variable intensidad competitiva del sector ejerce una influencia negativa pero moderada sobre las probabilidades de supervivencia del negocio. Una hipótesis que explicaría su menor importancia relativa, al igual que la de la variable potencial de localización, es que las características del emprendedor y el conocimiento del mercado permiten que el propietario o directivo actúen estratégicamente, gestionando los recursos organizacionales de manera que las restricciones impuestas por el mercado o por el espacio geográfico puedan ser adecuadamente subsanadas. De esta forma, aún en el caso de haber incursionado en un sector de negocios altamente competido, en una localización desventajosa, la destreza, y en su caso la perseverancia del emprendedor o del directivo serían los factores determinantes para que el negocio subsista. Cabe hacer mención que en el estudio sólo se aprecia la variable supervivencia, mas no la rentabilidad o el crecimiento del negocio, por lo que nos arroja información de un segmento productivo que se mantiene en el mercado aun cuando sus ganancias sean reducidas. También con viene aclarar que en la valoración del potencial de la localización no se consideraron el comercio electrónico, las redes sociales y páginas web que modifican la accesibilidad del negocio sin los costos de transporte. 
Al desagregar la variable Perfil Emprendedor y comparar los valores promedio obtenidos en cada submuestra se encontró que las características donde se observaron diferencias estadísticamente significativas son optimismo, motivación de logro, locus de control, innovación y autonomía, lo que nos permite apreciar que el componente actitudinal así como algunos rasgos de personalidad de los emprendedores y directivos juegan un papel relevante en la permanencia de las unidades de negocio. Cabe señalar que en los rasgos tolerancia al estrés y tolerancia al riesgo (que podrían estar más relacionadas con la rentabilidad y el crecimiento) no se observaron diferencias estadísticamente significativas.

Lo anterior podría dar una pauta para implementar programas para el desarrollo de emprendedores, en adición a la capacitación técnica que es necesario ofrecerles.

Finalmente, la variable concentración de competidores parece ejercer una influencia poco significativa en las probabilidades de supervivencia de este tipo de negocios.

Estos hallazgos nos permiten concluir que el desarrollo de las capacidades del emprendedor y la realización de estudios de mercado son cruciales para mejorar las probabilidades de sobrevivencia de una micro empresa.

Estudios posteriores pueden profundizar sobre estos hallazgos para determinar si son generalizables para empresas de mayor tamaño o que a través de la tecnología superan barreras del mercado y limitaciones de localización mediante herramientas de comercio electrónico.

\section{Referencias}

Bamiatzi, V.C.; Kirchmaier, T. (2014). Strategies for superior performance under adverse conditions: A focus on small and medium-sized high-growth firms. International Small Business Journal, 32(3): 259-284. http://dx. doi.org/10.1177/0266242612459534

Bruyat, Ch. and Julien, P. A. (2001). Defining the field of research in entrepreneurship. Journal of Business Venturing, 16 (2), 165. http://dx.doi.org/10.1016/S0883-9026(99)00043-9

Build Fabregà, M., \& Rocafort Nicolau, A. (2016). Emprendimiento y supervivencia empresarial en época de crisis: El caso de Barcelona. Intangible Capital, 12(1), 95-120. http://dx.doi.org/10.3926/ic.689

Covin, J.G. \& Slevin, D.P. (1989). "Strategic management of small firms in hostile and benign environments". Strategic Management Journal, 10, 75-87. https://doi.org/10.1002/smj.4250100107

Cowling, M. et al. (2014). What really happens to a small and medium-sized enterprises in a global economic recession? UK evidence on sales and job dynamics. International Small Business Journal, 33(5): 488-513. http:// dx.doi.org/10.1177/0266242613512513

De Juan-Vigaray, M. D. (2005). Comercialización y retailing. Pearson Educación. Disponible en https://www. pearsoneducacion.net/espa\%C3\%B1a/TiendaOnline/es-ebook-9788483222492- .

Diario Oficial de la Federación, del 23 de diciembre de 2011. REGLAS de Operación del Fondo de Apoyo para la Micro, Pequeña y Mediana Empresa (Fondo PyME) para el ejercicio fiscal 2012. Disponible en https://www. dof.gob.mx/nota_detalle.php?codigo=5226587\&fecha=23/12/2011 
Formichella (2002). "The Entrepreneurship Concept and Its Relation with the Education, the Employment and the Local Development”. National Institute of Farming Technology. Disponible en http://municipios.unq.edu.ar/ modules/mislibros/archivos/MonografiaVersionFinal.pdf y consultado el 20 de marzo de 2019.

Garrocho, C. (2003). La teoría de interacción espacial como síntesis de las teorías de localización de actividades comerciales y de servicios. Economía, Sociedad y Territorio, vol. IV, núm. 14, julio-diciembre, 2003, pp. 203-251, El Colegio Mexiquense, A.C. DOI: 10.22136/est002003426

Global Entrepreneurship Monitor (2015) APS Global Individual Level Data. Disponible en https://www.gemconsortium.org/data y consultado el 10 de febrero de 2018.

Grant, R.M. (1991). The Resource-Based Theory of Competitive Advantage: Implications for Strategy Formulation. California Managem. Rev., Spring, pp. 114-135. https://doi.org/10.2307/41166664

Grasland, C. (2004) “Interacción Espacial”. Hypergéo. Recuperado de http://www.hypergeo.eu/IMG/_article_ PDF/article_192.pdf, el 13 de abril de 2018.

Hornaday, J.A. (1982). "Research about living entrepreneurs", in K. C.A., S. D.L. y V. K.H. (Eds.), Encyclopedia of entrepreneurship (pp. 91-101). Englewood Cliffs, NJ: Prentice Hall.

INEGI (2015). Censos Económicos 2014. Resultados definitivos. Disponible en http://www.inegi.org.mx/est/contenidos/proyectos/ce/ce2014/default.aspx y consultado el 01 de febrero de 2018

INEGI (2018). Esperanza de vida de los negocios en México. Disponible en http://www.inegi.org.mx/inegi/contenidos/investigacion/Experimentales/Esperanza/default.aspx y consultado el 25 de abril de 2018.

Ibarra, S., y Suárez, J. (2002). La teoría de los recursos y las capacidades: un enfoque actual en la estrategia empresarial. Disponible en https://dialnet.unirioja.es/servlet/articulo?codigo=793552 y consultado el 01 de febrero de 2018

Martin, R.; Sunley, P. (2014). On the notion of regional economic resilience: Conceptualization and explanation. Journal of Economic Geography, 15(1): 1-42. Oxford University Press. http://dx.doi.org/10.1093/jeg/lbu015

Molina, R. (2009). La incidencia de las competencias esenciales y el propietario-dirigente en el fuerte crecimiento de la Pyme manufacturera en San Luis Potosí, México. Cuadernos de Administración. Bogotá, Colombia, 22 (38): 121-144, enero-junio de 2009, 121-144. Disponible en https://revistas.javeriana.edu.co/index.php/cuadernos_admon/article/view/3867 y consultado el 28 de marzo de 2018

Morales-Gualdrón, S. T., \& Zapata, U. P. (2015). Factores del perfil del emprendedor y de la gestión del servicio que inciden en la supervivencia empresarial: casos del oriente de Antioquia (Colombia). Pensamiento \& Gestión, (38), 176-207. http://dx.doi.org/10.14482/pege.38.7705

Moscote, O., \& Rincón, W. (2017). Logit and Probit Models: an application. Disponible en http://repository.usta. edu.co/handle/11634/6414 y consultado el 25 de marzo de 2018.

Pedrosa García, I. (2015). Evaluación de la personalidad emprendedora mediante un test adaptativo informatizado. Tesis doctoral, Universidad de Oviedo, España. Disponible en digibuo.uniovi.es/dspace/bitstream/10651/33327/1/TD_ignaciopedrosa.pdf y consultado el 18 de marzo de 2018.

Porter, M. E. (2008). Las cinco fuerzas competitivas que le dan forma a la estrategia. Harvard Business Review, 86(1), 58-77. http://dx.doi.org/10.4236/me.2011.23043

Rauch, A. y Frese, M. (2007). "Born to be an entrepreneur? Revisiting the personality approach to entrepreneurship" in J.R. Baum, M. Frese y R.J. Baron (Eds.), The psychology of entrepreneurship (pp. 41-65). Mahwah, NJ: Erlbaum. Disponible en https://www.rug.nl/research/portal/publications/born-to-be-an-entrepreneur-revisiting-the-personality-approach-to-entrepreneurship(a093eccf-bec5-423b-a087-905568fc1236)/export.html y consultado el 03 de marzo de 2019

Ruppenthal, T., \& Bausch, A. (2009). Research on export performance over the past 10 years: A narrative review. European Journal of International Management, 3(3), 328-364. doi:10.1504/ejim.2009.026995 
Sánchez, J., Ward, A., Hernández, B., y Florez, J. (2017). Educación emprendedora: Estado del arte. Propósitos y Representaciones, [S.1.], v. 5, n. 2, p. 401-473, oct. 2017. http://dx.doi.org/10.20511/pyr2017.v5n2.190.

Seth, N., Deshmukh, S. G., \& Vrat, P. (2005). Service quality models: a review. International Journal of Quality \& Reliability Management (Vol. 22, pp. 913-

949). doi:10.1108/02656710510625211

Shane, S. y Venkataraman, S. (2000). "The promise of entrepreneurship as a field of research". Academy of Management Review, 25(1), 217-226. doi:10.2307/259271

Vecchio, R.P. (2003). "Entrepreneurship and leadership: common trends and common threads”. Human Resource Management Review, 13, 303-327. https://doi.org/10.1016/S1053-4822(03)00019-6

White, J. C., Varadarajan, P. R., \& Dacin, P. A. (2003). Market situation interpretation and response: The role of cognitive style, organizational culture, and information use. Journal of Marketing, 67(3), 63-79. doi:10.1509/ jmkg.67.3.63.18654

Williams, N.; Vorley, T. (2014). Economic resilience and entrepreneurship: Lessons from the Sheffield City Region. Entrepreneurship \& Regional Development. https://doi.org/10.1080/08985626.2014.894129

Zou, S., \& Cavusgil, S. T. (2002). The GMS: A broad conceptualization of global marketing strategy and its effect on firm performance. Journal of Marketing, https://doi.org/10.1509/jmkg.66.4.40.18519 